\title{
MORBIDADE POR CÂNCER DE COLO UTERINO EM MULHERES DE RESERVA INDÍGENA NO MATO GROSSO DO SUL
}

Jackeline Camargos Pereira1 ${ }^{1}$ Fabiane Melo Heinen Ganassin², Roberto Dias de Oliveira ${ }^{3}$, Rogério Dias Renovato ${ }^{4}$, Elaine Aparecida Mye Takamatu Watanabe ${ }^{5}$

RESUMO: O objetivo deste estudo, ecológico transversal, foi conhecer a prevalência de lesões pré-cancerosas e cancerosas de colo uterino e a cobertura do rastreamento por meio do exame Papanicolaou em mulheres das aldeias Jaguapirú e Bororó, da Reserva Índigena de Dourados. Os dados foram coletados do Sistema de Informações de Saúde Indígena e do Programa de Controle do Câncer de Colo de Útero do Pólo-Base de Dourados/FUNASA, referentes ao período de 2004 a 2006. Os resultados demonstraram que o exame tem sido realizado mais em mulheres entre 15 e 34 anos; a cobertura do rastreamento aumentou na aldeia Jaguapirú e diminuiu na Bororó. Quanto às alterações citopatológicas, 5,7\% dos exames em mulheres da aldeia Jaguapirú indicaram lesões pré-malignas; na aldeia Bororó esse resultado foi 2,9\%. Evidenciou-se redução na gravidade das lesões cervicais no decorrer da implantação do programa de rastreamento. Estes achados indicam a importância dos programas de controle para a população estudada.

PALAVRAS-CHAVE: Neoplasias do colo do útero; Saúde da mulher; Saúde indígena.

\section{MORBIDITY FROM CERVICAL CANCER AMONG WOMEN ON AN INDIGENOUS RESERVE IN MATO GROSSO DO SUL}

\begin{abstract}
The objective of this ecological and transversal study was to learn about the prevalence of precancerous and cancerous lesions of the cervix and the coverage of screening with the cervical smear test among women in the villages of Jaguapirú and Bororó in the Indian Reserve of Dourados. Data was collected from the Indigenous Health Information Service and the Polo-Base de Dourados/FUNASA Program for the Control of Cervical Cancer, referent to the period 2004-2006. The results showed that the test had mostly taken place among women between 15 and 34 years of age. Screening coverage increased in Jaguapirú and decreased in Bororó. As for cytopathological changes, 5.7\% of tests in Jaguapirú indicated premalignant lesions; in the village of Bororó the result was $2.9 \%$. There was evidence of a reduction in the gravity of cervical lesions as the screening program went on. These findings show the importance of control programs for the population under study.
\end{abstract}

KEYWORDS: Uterine cervical neoplasms; Women's health; Indigenous health.

\section{MORBIDAD POR CÁNCER DE CUELLO UTERINO EN MUJERES DE RESERVA INDÍGENA EN MATO GROSSO DO SUL}

\begin{abstract}
RESUMEN: El objetivo de este estudio, ecológico transversal, fue conocer la prevalencia de lesiones precancerosas y cancerosas de cuello uterino y hacer la verificación por medio del examen Papanicolau en mujeres de las aldeas Jaguapirú y Bororó, de la Reserva Indígena de Dourados. Los datos fueron recogidos del Sistema de Informaciones de Salud Indígena y del Programa Control de Cáncer de Cuello de Útero del Polo-Base de Dourados/FUNASA, sobre el periodo de 2004 a 2006. Los resultados demostraron que el examen es realizado más en mujeres entre 15 y 34 años; la cobertura para rastrear creció en la aldea Jaguapirú y disminuyó en la Bororó. Acerca de las alteraciones citopatológicas, 5,7\% de los exámenes en mujeres de la aldea Jaguapirú apuntaron lesiones premalignas; en la aldea Bororó ese resultado fue 2,9\%. Se evidenció reducción en la gravedad de las lesiones cervicales a la medida que era hecha la implantación del programa para rastrear. Tales informaciones indican la importancia de los programas de control para la población estudiada.
\end{abstract} PALABRAS CLAVE: Neoplasias del cuello uterino; Salud de la mujer; Salud indígena.

\footnotetext{
${ }^{1}$ Enfermeira da Secretaria Municipal de Dourados/MS.

${ }^{2}$ Enfermeira. Mestre em Saúde Coletiva. Professora da Universidade Estadual de Mato Grosso do Sul - UEMS.

${ }^{3}$ Enfermeiro. Mestre em Doenças Infecciosas e Parasitárias. Professor do Curso de Graduação em Enfermagem da UEMS.

${ }^{4}$ Farmacêutico. Doutor em Educação. Professor do Curso de Graduação em Enfermagem da UEMS.

${ }^{5}$ Enfermeira. Mestre em Desenvolvimento Sustentável. Professora do Curso de Graduação em Enfermagem da UEMS.
}

Autor correspondente:

Rogério Dias Renovato

Universidade Estadual de Mato Grosso do Sul

R. Hilda Bergo Duarte, 296 - 79806-020 - Dourados-MS-Brasil

E-mail: rrenovato@uol.com.br

Recebido: 04/10/10 Aprovado: 22/02/11 


\section{INTRODUÇÃO}

O câncer de colo do útero é uma afecção iniciada com transformações intra-epiteliais progressivas que podem evoluir para um processo invasor num período que varia de 10 a 20 anos. É uma doença prevenível por meio da citopatologia oncótica e, graças à essa lenta progressão, é possível a detecção precoce de lesões pré-malignas ou malignas e o seu tratamento oportuno.

Nas duas últimas décadas, a literatura brasileira sobre saúde da mulher teve rápido crescimento e avanço com estudos que enfocam relações de gênero, saúde sexual, anticoncepção, epidemiologia de doenças sexualmente transmissíveis, câncer ginecológico, entre outros. Mas poucos estudos enfocam grupos étnicos/ raciais específicos, sendo que na sua maioria trabalham com mulheres negras. Assim, o tema da saúde da mulher indígena tem sido pouco investigado, e as escassas pesquisas realizadas são, em sua maioria, com etnias situadas dentro da Amazônia. Os estudos revelam

(...) um quadro alarmante, marcado por elevadas prevalências de doenças sexualmente transmissíveis, lesões ginecológicas de etiologia variada, mastopatias, além de queixas generalizadas de dores do baixo ventre, dispareunia e leucorréia ${ }^{(1: 9)}$.

Contudo, ao se considerar a diversidade étnicocultural dos povos indígenas não se pode generalizar os resultados dessas pesquisas para as demais comunidades/aldeias/etnias do Brasil ${ }^{(1)}$. O Instituto Sócio Ambiental estima que são 232 povos indígenas no Brasil $^{(2)}$. Dados estatísticos sobre esta população variam segundo as fontes de informação, em decorrência da falta de censo específico. De acordo com os dados disponíveis pelo Instituto Brasileiro de Geografia e Estatística, existe um total de 734.127 indígenas no Brasil, e destes, 350.829 são domiciliados em zona rural e 383.298 em zona urbana, perfazendo não mais que $0,5 \%$ da população brasileira ${ }^{(3)}$.

Esses povos estão distribuídos por todo o território nacional, e se dividem em várias etnias, com características sociais, culturais e econômicas distintas. Em Mato Grosso do Sul, há uma pluralidade de etnias: Chamacoco, Guarani Kaiowá, Guarani Ñandeva, Guató, Kadiwéu, Ofaié-Xavante e Terena ${ }^{(2)}$. No Município de Dourados, a Reserva Indígena (RID) contava, em 2004, com uma população de 10.393 indígenas, distribuídos em 3.539 hectares $^{(4)}$.

A RID é dividida em duas aldeias: Bororó, onde se destaca a presença maciça da etnia Ñandeva (que se autodenomina Guarani) e Kaiowá; e aldeia Jaguapirú, com população Terena e Guarani. Em ambas as aldeias se faz presente a figura dos mestiços. A divisão territorial da reserva é feita por uma linha hipotética traçada desde a sua implantação e respeitada pelos moradores. A RID está localizada na Zona Norte do Município de Dourados, distante 3,5 km do centro urbano e a $8 \mathrm{~km}$ do Município de Itaporã. Tem $13 \mathrm{~km}$ de extensão e é cortada pela rodovia MS-156 que liga Dourados a Itaporã.

Na RID, localiza-se o Hospital e Maternidade Porta da Esperança, uma instituição não-governamental, referência para o atendimento à saúde dos povos indígenas do Mato Grosso do Sul. Conta ainda com o Centro de Recuperação Nutricional para a população infantil indígena, bem como de serviço laboratorial. Na Aldeia Bororó situa-se o Posto de Saúde Ireno Isnard, e na Aldeia Jaguapirú uma Unidade Saúde da Família $^{(5)}$.

A saúde indígena em Dourados integra o Distrito Sanitário Especial Indígena do Mato Grosso do Sul, sendo um dos seis pólos-base, além de Aquidauana, Bonito, Amambai, Iguatemi e Campo Grande ${ }^{(6)}$. O Pólo-Base Dourados abrange, além da RID, composta pelas aldeias Bororó e Jaguapiru, as aldeias de Porto Cambira, Panambizinho, Panambi e Sucuriú.

Assim, a proposta deste estudo foi verificar a prevalência de câncer cérvico-uterino e de lesões precursoras do câncer do colo do útero em mulheres da reserva indígena de Dourados-MS, no período de 2004 a 2006, como também conhecer a cobertura das ações de detecção precoce do câncer do colo do útero na mesma população.

\section{METODOLOGIA}

Estudo ecológico transversal, com análise de dados secundários do Sistema de Informações de Saúde Indígena (SIASI) e do Programa Controle do Câncer do Colo de Útero (PCCCU) do Pólo-Base de Dourados/FUNASA, sobre exames citopatológicos realizados entre 2004 e 2006. Dados sobre lesões cancerosas foram buscados no Livro de Registros de Controle de Câncer do Colo de Útero do Centro de Atendimento à Mulher (CAM), do Município de Dourados/MS.

Foram incluídos neste estudo todos os dados disponíveis dos agregados populacionais referentes às mulheres indígenas das Aldeias Jaguapirú e Bororó 
da RID, com idade acima de 12 anos, dentro do período estudado. Segundo dados do SIASI, o número de mulheres acima de 12 anos na Aldeia Bororó foi de 1.322, 1.356 e 1.345, nos anos 2004, 2005 e 2006, respectivamente. Na Aldeia Jaguapirú foi de 1.525, 1.696 e 1.780, na mesma sequência cronológica. Também foram coletados dados pertinentes à população feminina da RID: número de exames por faixa etária, resultados com alterações patolológicas (HPV, NIC I, NIC II, NIC III) e lesões cancerosas, e dados relacionados à detecção do câncer de colo do útero.

Os dados foram armazenados no programa Excel, sendo calculadas as taxas de prevalência por período e prevalência anual de alterações citopatológicas, por aldeia, do período compreendido entre 2004 e 2006. Ao realizar a prevalência por período, a população foi ajustada para o meio do período (ano 2005), devido ao fato daquele ano apresentar maior contingente populacional.

Foi estimada a cobertura do rastreamento de lesões precursoras de câncer cérvico-uterino, considerando o número de exames citopatológicos realizados anualmente, em relação ao número de mulheres que deveriam ser atingidas. A cobertura do PCCCU foi estimada neste estudo uma vez que não se pode concluir que os exames foram realizados por mulheres diferentes.

Esta pesquisa foi encaminhada ao Comitê de Ética em Pesquisa da Universidade Federal de Mato Grosso do Sul, solicitando autorização de acordo com a Resolução n. 196 do Conselho Nacional de Saúde, tendo protocolo de aprovação n. 874, no dia 13 de março de 2007. Foi solicitado dispensa do Termo de
Consentimento Livre e Esclarecido - TCLE por ser um estudo de caráter retrospectivo e com utilização de dados secundários. As lideranças indígenas locais tiveram ciência do estudo; também foi obtida autorização da FUNAI e FUNASA para a realização da pesquisa. As instituições detentoras dos dados (CAM e FUNASA) autorizaram a coleta dos dados e a elas foi emitido o Termo de Compromisso para Utilização de Informações do Banco de Dados.

\section{RESULTADOS}

No período de 2004 a 2006 foram coletadas 999 amostras de citopatologia oncótica na Aldeia Jaguapirú e 278 na Bororó. A população feminina dessas aldeias, ajustada para o meio do período, são respectivamente 1.780 e 1.345 mulheres acima de 12 anos. A estimativa de cobertura do exame citopatológico no ano de 2004 na Aldeia Jaguapiru foi de 13,9\%, enquanto que na Aldeia Bororó foi de $8,5 \%$. Em relação ao ano 2005, na Aldeia Jaguapiru, verificou-se aumento de cobertura para $21,7 \%$, e para $23,5 \%$ em 2006. Já na Aldeia Bororó ocorreu o inverso, com redução da cobertura de 7,2\% em 2005 para 5,1\% em 2006.

Como a forma de registro de PCCCU do PóloBase de Dourados foi modificada em 2005 - até então não constava a idade das mulheres que realizavam a coleta - as informações da cobertura, por faixa etária, são relativas apenas aos anos 2005 e 2006. Assim, verificou-se que o exame preventivo foi mais realizado por mulheres jovens, entre 15 e 34 anos, em ambas as aldeias, como descritos nas figuras 1 e 2 .

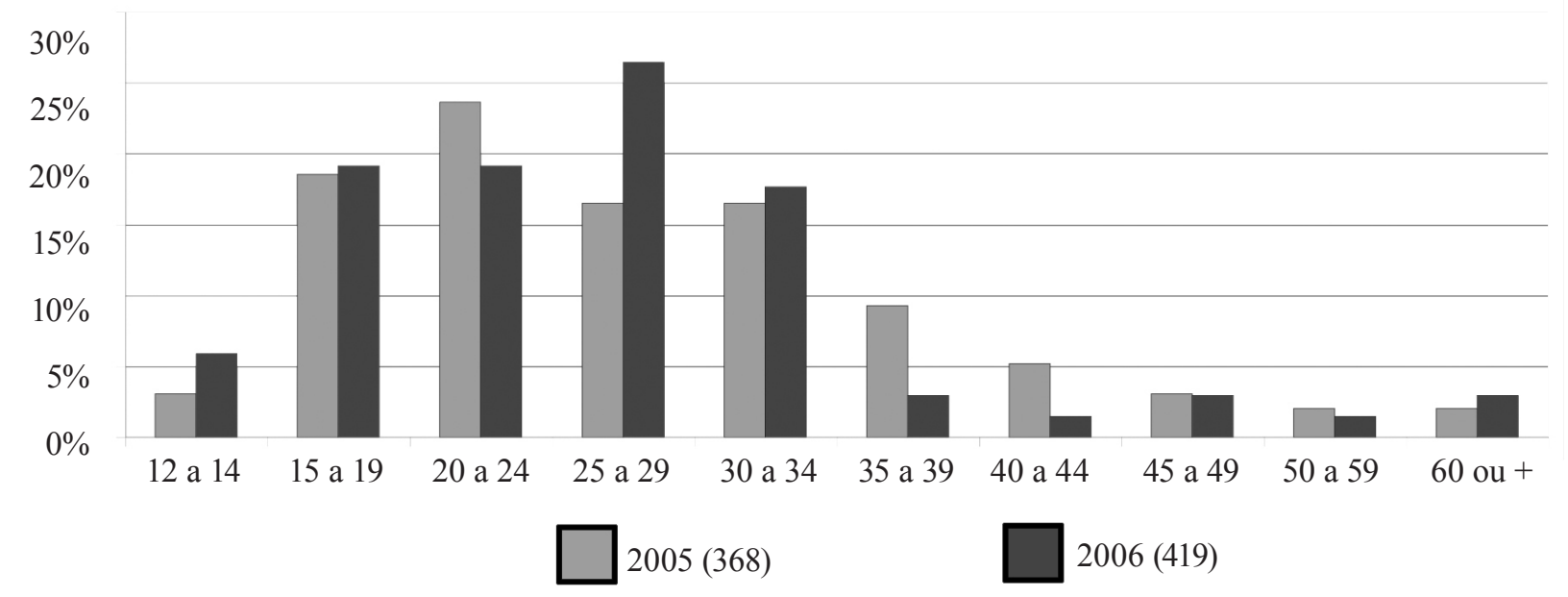

Figura 1 - Percentual de exames preventivos de câncer do colo do útero, segundo faixa etária e ano de coleta, na Aldeia Jaguapirú. Dourados/MS, 2005 e 2006 


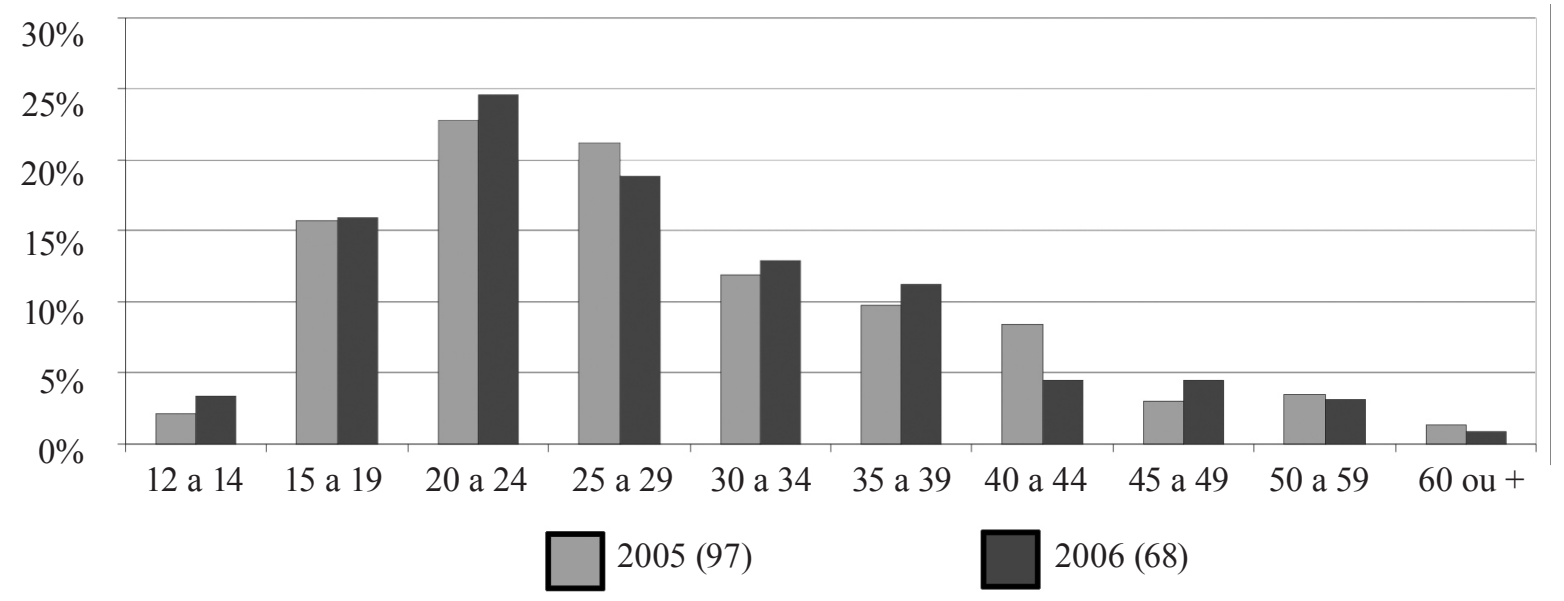

Figura 2 - Percentual de exames preventivos de câncer do colo de útero, segundo faixa etária e ano de coleta, na Aldeia Bororó. Dourados/MS, 2005 e 2006

Para o intervalo de idade de 35 a 49 anos, em 2005, a cobertura foi de 28,9\% na Aldeia Jaguapirú, e para o ano de 2006 teve uma ligeira redução, perfazendo $28,6 \%$. Esses valores superam dados nacionais, que, em 2002, mostraram uma cobertura estimada de $16,2 \%{ }^{(7)}$. Já na Aldeia Bororó, a cobertura para a referida faixa etária manteve-se baixa no ano de 2005 , com um percentual de $8,13 \%$, e diminuiu ainda mais em 2006, atingindo apenas $2,2 \%$ de cobertura.

Da mesma forma, o rastreamento da faixa etária de 25 a 59 anos, na Aldeia Bororó, tem sido baixo e apresentou queda acentuada no decorrer dos anos, com $10,76 \%$ em 2005 e de $6,69 \%$ em 2006. Houve um aumento na cobertura desse grupo etário na Aldeia Jaguapirú, perfazendo $31,19 \%$ de cobertura no ano de 2005; no ano de 2006 houve um leve aumento, alcançando $32,08 \%$ de cobertura.

Das coletas realizadas em mulheres da Aldeia Jaguapirú, no período de 2004 a 2006, 5,7\% (n=278) dos exames apresentaram alterações citopatológicas pré-malignas, sendo que $2,6 \%$ foi de HPV; $2,5 \%$ NIC I; $0,2 \%$ NIC II; e $0,4 \%$ NIC III. Na Aldeia Bororó esse índice foi de $2,88 \%$, entre estes $0,72 \%$ para HPV; $1,08 \%$ NIC I; $1,08 \%$ NIC II; e zero NIC III.

A distribuição dos resultados dos exames citopatológicos, conforme o tipo de lesão, por ano, para ambas as aldeias, está apresentada nas tabelas 1 e 2; nas quais percebe-se mudanças no perfil das lesões. No decorrer dos anos estudados houve diminuição no percentual de lesões de alto grau (NIC II e NIC III) e aumento nas de baixo grau (HPV e NIC I).
Tabela 1 - Percentual de exames citopatológicos alterados, segundo ano de realização, na Aldeia Jaguapirú. Dourados/ MS, 2004 a 2006

\begin{tabular}{lccc}
\hline & $\mathbf{2 0 0 4}$ & $\mathbf{2 0 0 5}$ & $\mathbf{2 0 0 6}$ \\
& $\mathbf{( 2 0 2 )}$ & $\mathbf{( 3 6 8 )}$ & $\mathbf{( 4 1 9 )}$ \\
\hline HPV & 0,94 & 2,72 & 3,34 \\
NIC I & 0,47 & 3,80 & 2,39 \\
NIC II & 0,47 & 0,27 & 0,00 \\
NIC III & 0,94 & 0,27 & 0,24 \\
\hline
\end{tabular}

Tabela 2 - Percentual de exames citopatológicos alterados, segundo ano de realização, na aldeia Bororó, Dourados/ MS, 2004 (113), 2005 (97) e 2006 (68)

\begin{tabular}{lccc}
\hline & $\mathbf{2 0 0 4}$ & $\mathbf{2 0 0 5}$ & $\mathbf{2 0 0 6}$ \\
& $\mathbf{( 1 1 3 )}$ & $\mathbf{( 9 7 )}$ & $\mathbf{( 6 8 )}$ \\
\hline HPV & 0,00 & 0,00 & 2,94 \\
NIC I & 0,00 & 1,03 & 2,94 \\
NIC II & 0,88 & 2,06 & 0,00 \\
NIC III & 0,00 & 0,00 & 0,00 \\
\hline
\end{tabular}

Não foram diagnosticadas lesões cancerosas em indígenas da RID, pelo exame Papanicolaou. Os casos positivos de carcinoma e adenocarcinoma foram diagnosticados por biópsia. Os registros sobre esse procedimento foram coletados no Centro de Atendimento à Mulher (CAM).

Também, foi diagnosticado um caso de carcinoma invasor em 2005, e outro em 2006, ambos na Aldeia Jaguapirú, o que corresponde a $0,12 \%$ da população feminina acima de 12 anos, no período de 2004 a 2006. 


\section{DISCUSSÃO}

Observa-se que a estimativa de cobertura do exame Papanicolaou foi maior na Aldeia Jaguapirú, onde residem as etnias Guarani-Ñandeva e Terena. Houve uma progressão, de 2004 para 2006, em 98\% no número de coletas realizadas nessa aldeia.

Ao contrário dos resultados da Aldeia Jaguapirú, na Aldeia Bororó o percentual de cobertura foi muito aquém do estabelecido, e o número de coletas realizadas diminuiu 40\% de 2004 para 2006. Nessa aldeia residem as etnias Kaiowá e Ñandeva. Em estudo sobre educação em saúde na Aldeia Bororó, confirmou-se que é comum a resistência dos indigenas Kaiowá em participar dos programas de prevenção, como campanhas de imunização e exame preventivo de câncer do colo uterino ${ }^{(4)}$.

Em relação à imunização, a resistência se deve ao uso de materiais perfurantes no corpo; e, em relação ao exame preventivo de câncer do colo uterino, além da resistência, as que se submetem a essa assistência não compreendem a importância do procedimento, deixando muitas vezes de buscar os resultados ${ }^{(5)}$.

A etnia Kaiowá manteve-se "reclusa" de influências da cultura ocidental, preservando suas características próprias, que sempre foram prioridades por parte dos líderes e pais de família. É interessante lembrar que o poder político, nesse grupo, é diretamente relacionado com as lideranças religiosas, de forma que há resistência ao modelo de saúde biomédico. A localização territorial da Aldeia Bororó também favoreceu a reclusão, pois esse grupo sempre residiu na parte da reserva mais afastada da Cidade de Dourados, o que dificultou o acesso tanto à cidade quanto às informações relacionadas à saúde. Também existe a falta de material didático e informativo específicos para a educação em saúde da população indígena, o que contribui para o fracasso de informações sobre a prevenção de doenças nessas populações ${ }^{(5)}$.

O Ministério da Saúde estabelece que o exame colpocitológico seja realizado em mulheres de 25 a 59 anos de idade, uma vez por ano e, após dois exames consecutivos com resultados negativos, a cada três anos. Ainda, estabelece que faixa etária prioritária para rastreamento seja dos 35 aos 49 anos, período que corresponde ao pico de incidência das lesões precursoras e que antecede o pico de mortalidade pelo câncer ${ }^{(7-8)}$. Em revisão de literatura sobre os fatores determinantes da cobertura do exame de Papanicolaou no Brasil verificou-se que mulheres da população geral e da faixa etária menor de 20 anos e maior de 50 anos submetem-se menos ao exame. Esses achados são similares aos do presente estudo, em relação às mulheres indígenas de faixa etária maior ${ }^{(9)}$.

Nesta pesquisa, observou-se que as mulheres indígenas mais jovens foram as que mais aderiram ao rastreamento. Tratando-se de comunidades indígenas, pode-se inferir que as jovens estão mais em contato com a sociedade do que em relação às mulheres mais velhas, e portanto, recebem mais informações sobre saúde e prevenção de doenças.

Em mulheres indígenas de Rondônia, etnias Suruí e Cinta Larga, o percentual dos exames citopatológicos também foi maior em mulheres jovens, na faixa etária de 16 a 25 anos, com $36,1 \%$ e $26,9 \%$, respectivamente ${ }^{(10)}$. Outro estudo realizado em índias da tribo Parakanã, no sudoeste do Estado do Pará, para detecção de cérvico-vaginites e lesões precursoras do câncer do colo uterino, entre as lesões intra-epiteliais de baixo grau encontraram 23,2\% para HPV e 1,4\% NIC I. Entre as lesões intra-epiteliais de alto grau NIC II, NIC III e carcinoma invasor, os resultados apontaram $1,4 \%{ }^{(11)}$.

Em outra pesquisa com a finalidade de rastrear o câncer do colo uterino em indígenas do Parque Indígena do Xingu, no norte do Estado do Mato Grosso, entre 1989 e 1996, as lesões intra-epiteliais de baixo grau encontradas foram 2\% para HPV e 1\% NIC I; entre as lesões intra-epiteliais de alto grau NIC II, NIC III e carcinoma invasor, os resultados apontaram $1 \%$ em todos ${ }^{(12)}$.

Os índices de HPV encontrados entre indígenas da Aldeia Jaguapirú (2,6\%) são maiores que em outras aldeias indígenas do Brasil, exceto da tribo Parakanã ${ }^{(11)}$. Já em mulheres da Aldeia Bororó, as taxas de HPV $(0,7 \%)$ foram relativamente baixas em comparação às indígenas de outras localidades. Segundo dados do Sistema de Informação de Controle do Colo do Útero - SISCOLO ${ }^{(7)}$, o percentual de HPV por exame scitopatológicos realizados no Brasil entre janeiro e julho de 2002 foi de $0,7 \%$.

A infecção pelo HPV é tida como principal fator de risco para o surgimento de lesões precursoras e do próprio câncer cérvico-uterino. Considerando que este patógeno é com freqüência sexualmente transmitido, podemos inferir que as mulheres da RID vêm adquirindo esses patógenos por relação sexual. Embora não se possa afirmar o que está provocando estes índices na RID, especialmente da Aldeia Jaguapirú, existe a possibilidade deste fator estar ligado a maior proxi- 
midade desta população indígena com a sociedade não-indígena.

As taxas de lesões intra-epiteliais de baixo grau (NIC I) também são maiores em relação a algumas comunidades indígenas ${ }^{(10-12)}$ e ao percentual nacional ${ }^{(7)}$. Displasias moderadas (NIC II) tiveram baixa incidência na Aldeia Jaguapirú, porém elevadas na Bororó. O índice de lesão de alto grau de malignidade (NIC III) foi baixos na Aldeia Jaguapirú e nulo na Bororó.

O aumento na cobertura do exame preventivo na Aldeia Jaguapirú, durante os anos estudados, parece sugerir uma relação com a redução nos índices de lesões de alto grau. Esta evidência demonstra que o resultado do trabalho preventivo identifica lesões ainda em estágios de baixo grau.

\section{CONSIDERAÇÕES FINAIS}

O quadro de saúde dos povos indígenas está diretamente relacionado a processos históricos de mudanças sociais, econômicas e ambientais. Também a influência da cultura no comportamento do ser humano é incontestável, sendo necessário considerar a maneira como as pessoas explicam as causas das enfermidades, os tipos de tratamento em que acreditam e a quem recorrem, assim como o que fazem para evitar as doenças.

O presente estudo procurou conhecer a prevalência de lesões pré-cancerosas e cancerosas de colo uterino em indígenas da RID, bem como a cobertura de ações de detecção precoce dessa patologia. Observou-se aumento na cobertura do exame preventivo na Aldeia Jaguapirú durante os anos estudados. Concomitante a esse aumento, houve, em ambas as aldeias, redução nos índices de lesões de alto grau. Apesar da resistência das mulheres em realizar o exame, observou-se maior aderência das mulheres indígenas com menos de 35 anos de idade, ou seja, as que se encontram na faixa etária de menor risco.

Os comportamentos de prevenção são baseados nos conhecimentos e crenças dos sujeitos sobre a doença, determinando assim, o modo como esses compreendem o câncer, sua etiologia, a importância e as formas de prevenção. Isso pode explicar a melhora na cobertura do rastreamento, considerando que as ações voltadas à saúde da mulher começaram a ser difundidas no contexto das aldeias indígenas há seis anos, de forma que a população indígena passou a ter mais informações sobre a própria saúde.
Ao contrário dos resultados da Aldeia Jaguapirú, na Aldeia Bororó a cobertura foi baixa e diminuiu no decorrer dos anos. A compreensão e a aceitabilidade da concepção saúde e doença daquela população, em especial a da etnia Kaiowá, são essenciais para desencadear maior eficácia de programas de saúde, principalmente o de prevenção do câncer de colo uterino.

Como exposto anteriormente, não são apenas as crenças que influenciam o comportamento preventivo, mas também o conhecimento sobre a detecção precoce, o tratamento e formas de prevenção. Desse modo, a utilização de materiais didáticos e informativos específicos para a educação em saúde da população indígena, no idioma das etnias e, principalmente, com motivos contextualizados, pode contribuir para a disseminação de conhecimentos nas comunidades indígenas.

\section{REFERÊNCIAS}

1. Coimbra Júnior CEA, Garnelo Luiza. Questões de saúde reprodutiva da mulher indígena no Brasil. In: Monteiro S. Sansone L. Etnicidade na América Latina: um debate sobre raça, saúde e direitos reprodutivos. Rio de Janeiro: FIOCRUZ; 2004. p.15373.

2. Ricardo B, Ricardo F, editores. Povos indígenas no Brasil: 2001-2005. São Paulo: Instituto Socioambiental; 2006.

3. IBGE. Instituto Brasileiro de Geografia e Estatística. Tendências demográficas: uma análise dos indígenas com base nos resultados da amostra dos censos demográficos 1991 e 2000. Rio de Janeiro: IBGE; 2007.

4. Jesus DL. A transformação da reserva indígena de Dourados - MS em território turístico: valorização sócio-econômica e cultural [dissertação]. Dourados (MS): Universidade Federal de Mato Grosso do Sul; 2004.

5. Troquez MCS. Educação em saúde na aldeia Bororó: o índio Kaiowá de Dourados (MS) [dissertação]. São Carlos (SP): Universidade Federal de São Carlos; 2002.

6. Fundação Nacional de Saúde. Atenção à saúde dos povos indígenas: caracterização e estágio da implantação dos distritos sanitários especiais indígenas; 1999 [acesso em 23 set 2007]. Disponível: 
www.bvsde.paho.org/bvsapi/p/fulltext/distritos/ matogrosso.pdf

7. Ministério da Saúde (BR). Secretaria de assistência a saúde. Instituto Nacional de Câncer. Viva Mulher. Câncer do colo do útero: informações técnicogerencias e ações desenvolvidas. Rio de Janeiro: INCA; 2002.

8. Oliveira SL, Almeida ACH. A percepção das mulheres frente ao exame de Papanicolaou: da observação ao entendimento. Cogitare Enferm. 2009;14(3):518-26.

9. Martins LFL, Thuler LCS, Valente JG. Cobertura do exames de Papanicolaou no Brasil e seus fatores determinantes: uma revisão sistemática da literatura. Rev Bras Ginecol Obstet 2005;27(8):485-92.

10. Rodrigues DE, Escobar AL, Marques RC. Prevalência do câncer cérvico-uterino em mulheres indígenas Suruí e Cinta Larga, Rondônia, Brasil. 2007 [acesso em 11 mar 2007]. Disponível: www.unir.br/html/pesquisa/ Pibic_XIV/pibic2006/arquivos/Areas/Vida\%20e\%20 Saude/html/Daiana\%20Evangelista.html

11. Brito EB, Menezes RC, Martins SJ, Bastos MG, Sousa A. Estudo preliminar para detecção de cérvicovaginites e lesões precursoras do câncer de colo uterino em índias da tribo Parakanã. Rev Assoc Med Bras 1996;42(1):11-15.

12. Taborda WC. Rastreamento do câncer de colo uterino em índias do parque indígena do Xingu, Brasil central. Rev Panam Salud Publica. 2000;7(2):92-6. 